TITLE:

\title{
Non-Local Fokker-Planck Equation of Imperfect Impulsive Interventions and its Effectively Super-Convergent Numerical Discretization
}

\section{$\operatorname{AUTHOR}(\mathrm{S})$ :}

Yoshioka, Hidekazu; Yaegashi, Yuta; Tsujimura, Motoh; Fujihara, Masayuki

\section{CITATION:}

Yoshioka, Hidekazu ... [et al]. Non-Local Fokker-Planck Equation of Imperfect Impulsive Interventions and its Effectively Super-Convergent Numerical Discretization.

Communications in Computer and Information Science 2019, 1094: 79-91

\section{ISSUE DATE:}

2019-10

URL:

http://hdl.handle.net/2433/254135

\section{RIGHT:}

This is a post-peer-review, pre-copyedit version of an article published in Communications in Computer and Information Science. The final authenticated version is available online at: http://dx.doi.org/10.1007/978-981-15-10786_7.; The full-text file will be made open to the public on 21 October 2020 in accordance with publisher's 'Terms and Conditions for Self-Archiving'.; この論文は出版社版でありません。引用の際には出版社版をご確認ご利用ください。; This is not the published version. Please cite only the published version. 


\title{
Non-Local Fokker-Planck Equation of Imperfect Impulsive Interventions and Its Effectively Super- convergent Numerical Discretization
}

\author{
Hidekazu Yoshioka1 [0000-0002-5293-3246], Yuta Yaegashi², Motoh Tsujimura ${ }^{3 \text { [0000-0001-6975- }}$ \\ ${ }^{9304]}$ and Masayuki Fujihara ${ }^{2}$ \\ ${ }^{1}$ Shimane University, Nishikawatsu-cho 1060, Matsue 690-8504, Japan \\ yoshih@life.shimane-u.ac.jp \\ ${ }^{2}$ Kyoto University, Kitashirakawa Oiwake-cho, Kyoto 606-8502, Japan \\ yaegashi.yuta.54s@st.kyoto-u.ac.jp \\ fujihara@kais.kyoto-u.ac.jp \\ ${ }^{1}$ Doshisha University, Karasuma-Higashi-iru, Imadegawa-dori, Kyoto 602-8580, Japan \\ mtsujimu@mail.doshisha.ac.jp
}

\begin{abstract}
Human interventions to control environmental and ecological system dynamics are efficiently described as impulsive interventions by which the system state suddenly transits. Such interventions in applications are imperfect in the sense that the state transition is not exactly controllable and thus uncertain. Mathematical description of the imperfect impulsive interventions, despite relevance in practical problems of environmental and ecological engineering, has not been addressed so far to the best of the authors' knowledge. The objectives and contributions of this research are formulation and numerical computation of single-species population dynamics controlled through imperfect impulsive interventions. We focus on a management problem of a waterfowl population as a model problem where the population dynamics follows a stochastic differential equation subject to impulsive harvesting. We show that the stationary probability density function of the population dynamics is governed by a 1-D Fokker-Planck equation with a special non-locality, which potentially becomes an obstacle in analyzing the equation. We demonstrate that the equation is analytically solvable under a simplified condition, which is validated through a Monte-Carlo simulation result. We also demonstrate that a simple finite volume scheme can approximate its solution in a stable, conservative, and super-convergent manner.
\end{abstract}

Keywords: Imperfect Impulsive Intervention, Non-local Fokker-Planck Equation; Exponentially-fitted Finite Volume Scheme

\section{Introduction}

Human interventions are becoming more common in management of environmental and ecological system dynamics, such as extermination of invasive species [1] and harvesting regulation of biological resources [2]. Establishment of management policies of the system dynamics from the viewpoint of both cost-effectiveness and feasibility is 
an indispensable topic. To this end, we incorporate the imperfect interventions into the management problems because the human interventions do not always progress just as planned. The imperfectness is caused by uncertainties with respect to the magnitude of interventions.

The dynamics of environmental and ecological systems is described by stochastic differential equations (SDEs) [3], and management problems through human interventions to the system dynamics are described as stochastic control problems [4,5]. Pesticide applications for exterminating natural enemies of crops [6] and harvesting of bird population as a predator of fishery resources [7-8] are such examples. The impulse control approach provides a suitable analytical method if the intervention causes a discrete change in the system. Impulse control problems arise not only in environmental and ecological management problems but also in many other engineering research areas [910]. The optimal intervention policies to control system dynamics have been mathematically studied in detail from the viewpoint of the optimality equations [9]. However, much less attentions have been paid for behavior of the controlled system dynamics.

A Fokker-Planck equation is a governing differential equation of a probability density function (PDF) of stochastic system dynamics [11]. Recently, Yaegashi et al. [12] approached this issue through derivation and analysis of a 1-D Fokker-Planck equation of the controlled single-species population dynamics, which is the governing differential equation of the PDF of the state variable. However, they assumed that human interventions are perfect and that there exists no uncertainty in the interventions, which is not consistent with the above-mentioned reality of environmental and ecological systems management. This motivates us to extend their approach to imperfect interventions, which is a topic not addressed so far to the best of the authors' knowledge.

The objectives and contributions of this research are formulation and numerical computation of single-species population dynamics controlled by imperfect impulsive interventions. A particular emphasis is put on the fish-eating waterfowl management problem [7, 8]: a serious ecological problem in many countries of Europe, America, Asia, and New Zealand. This is an engineering problem with a relatively simple dynamics following an SDE subject to impulsive harvesting [8, 12]. We show that the stationary PDF of the population is governed by a 1-D Non-Local Fokker-Planck Equation (NL-FPE) with a special non-locality, which is potentially an obstacle in its analysis and computation. We demonstrate that the NL-FPE is analytically solvable in a simplified case. The solution is physically validated through a Monte-Carlo simulation. We develop an exponentially-fitted (local exact solution-based) finite volume scheme to discretize the NL-FPE in a stable, conservative, and super-convergent manner; the last one implies second-order convergence despite the equation has an advection term.

\section{Mathematical Model}

\subsection{Impulse Control Model}

Controlled population dynamics. A long-term model stochastic impulse control problem for population management is reviewed [8]. The decision-maker managing the population dynamics can impulsively reduce the population through interventions, which 
are carried out in a much shorter timescale than that of the dynamics. The population in a habitat at time $t \geq 0$ is denoted as $X_{t} \geq 0$, which is right continuous with left limits as in the conventional impulse control models [10]. The population dynamics, namely temporal evolution of the process $\left(X_{t}\right)_{t \geq 0}$, is assumed to be governed by the Itô's SDE subject to interventions:

$$
\left\{\begin{array}{c}
\mathrm{d} X_{t}=X_{t}\left(\mu \mathrm{d} t+\sigma \mathrm{d} B_{t}\right), \tau_{i} \leq t<\tau_{i+1} \\
X_{\tau_{i}}=X_{\tau_{i-}}-\zeta_{i}
\end{array}\right.
$$

with the initial population $X_{0-}=x \geq 0$, where $\mu>0$ is the deterministic growth rate, $\sigma>0$ is a volatility with $2 \mu>\sigma^{2}$, and $B_{t}$ is the 1-D standard Brownian motion on a usual complete probability space [3]. The second line of (1) is the human interventions, where each $\tau_{i}\left(i=0,1,2, \cdots, \tau_{0}=0\right)$ is the stopping time adapted to a natural filtration generated by the Brownian motion and represents the time at which the population is harvested, and $\zeta_{i}\left(i=0,1,2, \cdots, \zeta_{0}=0\right)$ is the magnitude of harvesting at $\tau_{i}$.

Human interventions $\eta=\left(\tau_{i}, \zeta_{i}\right)_{i=0,1,2, \ldots}$ to control the population dynamics (1), which is hereafter simply referred to as the policy, are determined so that a performance index considering both costs and benefits of the population management is minimized. The performance index $J=J(x ; \eta)$ is a functional of the process $\left(X_{t}\right)_{t \geq 0}$ and a policy $\eta$. The performance index in this model is [7-8]

$$
J(x ; \eta)=\mathrm{E}\left[\int_{0}^{\infty} e^{-\delta s}\left(-u X_{s}^{M}+d X_{s}^{m}\right) \mathrm{d} s+\sum_{i=1}^{\infty} e^{-\delta \tau_{i}} K\left(\zeta_{i}\right)\right],
$$

where $\mathrm{E}$ is the expectation, $\delta>0$ is a sufficiently large discount rate as an inverse of the time-scale of the decision-making, $u>0, d>0,0<M<1$ and $m>1$ are constants. The first term in the right-hand side of (2) is the discounted sum of the utility $\left(-u X_{s}^{M}\right)$ and disutility $\left(d X_{s}^{m}\right)$ that the population may provide during the management period. Notice that the disutility is considered to be a non-negative value because we will consider a minimization problem. As explained in Yaegashi et al. [7], this term gives a simplest lumped representation of the sum of the utility and disutility. The sum $-u X_{s}^{M}+d X_{s}^{m}$ is convex and unimodal, and thus takes a global minimum value with some positive $X_{s}>0$. Its minimizer is considered as a desired state of the population to be achieved through human interventions. The second term of (2) is the discounted sum of the cost of each harvesting, where

$$
K(\zeta)=k_{1} \zeta+k_{0}(\zeta>0) .
$$

Here, $k_{1}>0$ is the proportional cost and $k_{0}>0$ is the fixed cost.

Following the standard methodology for impulse control problems [13, 14], the following threshold type strategy turns out to be optimal:

\section{(Optimal policy)}


There exists the two thresholds $\bar{X}$ and $\underline{x}(0<\underline{x}<\bar{X})$ whose values depend on values of the model parameters. At each time $t \geq 0$, if $X_{t_{-}}<\bar{x}$, then no intervention is taken If $X_{t-} \geq \bar{x}$, then the intervention is immediately taken and $X_{t-}$ is reduced to $\underline{x}$.

In this way, the optimal policy is characterized with the thresholds $\bar{x}$ and $\underline{x}$, and the population is confined in a compact interval $[0, \bar{x}]$ and is harvested only when the population hits the upper threshold $\bar{x}$.

Imperfect interventions. A drawback of the above-presented model is that the human interventions are achieved exactly in the sense that paying the cost $K(\zeta)$ reduces the population by the amount of $\zeta$. However, it is plausible to assume that such an exact intervention is not realized in practice, but there exists uncertainty between the cost and realization. Mathematically, this means that paying the cost $K(\zeta)$ reduces the population, but the effectively reduced population is $Z \zeta$ not $\zeta$, where $Z>0$ is a stochastic variable that is not predictable for the decision-maker. This means that the population truly reduced at $\tau_{i}$ is $(\bar{x}-\underline{x}) Z_{i}$ because of the imperfectness of interventions, which equals to the perfect model only if $Z_{i}=1$. In what follows, we call an optimal control with the uncertainty $Z_{i}$ as an imperfect intervention.

Hereafter, for the sake of brevity of descriptions, we set the amount of reduced population at $\tau_{i}$ by the imperfect optimal control as $\bar{X}-Z_{i}$ with an abuse of notation, again it equals $\bar{x}-\underline{x}$ if $Z_{i}=\underline{x}$. We assume that each $Z_{i}(i=0,1,2, \ldots)$ is possibly related to $\underline{x}$, and is i.i.d. Furthermore, each $Z_{i}$ is assumed to be generated by a PDF $q=q(z)$ of $z=Z$ supported on $[a, b]$ such that $0<\bar{X}-Z_{i}<\bar{X}$. Fig. 1 plots a sample path of the controlled process $\left(X_{t}\right)_{t \geq 0}$ by an imperfect intervention. Note that the impulsive intervention itself is deterministic here, and is different from the unpredictable ones that reset the process randomly [15].

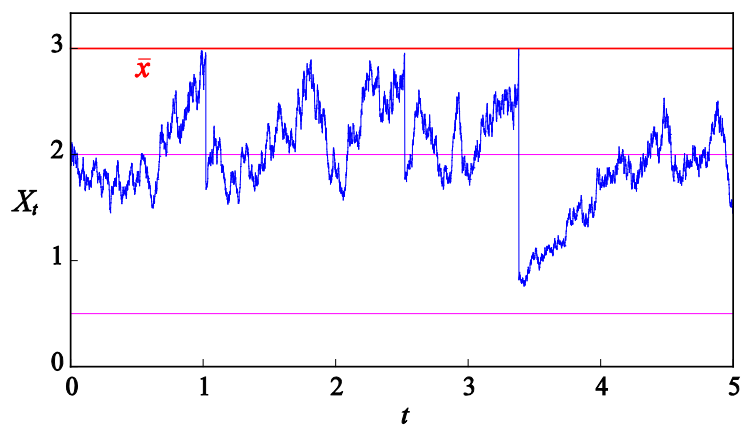

Fig. 1 A sample path of the controlled population dynamics (blue), the upper threshold $\bar{x}$ (red), and the uncertainty range $[a, b]$ (pink). 


\subsection{Non-local Fokker-Planck Equation}

Derivation. The PDF of the controlled process $\left(X_{t}\right)_{t \geq 0}$ under imperfect interventions is denoted as $p(y)$ for $0 \leq y \leq \bar{x}$, where $y$ represents the current value of the population. In general, a Fokker-Planck equation governs a PDF [11], and the equation is given as a conservative elliptic or parabolic differential equation subject to appropriate initial and/or boundary conditions. These equations are local like the conventional heat and wave equations. However, Yaegashi et al. [12] theoretically showed for the perfect impulse control model that the Fokker-Planck equation has a non-local term connecting the probability flux at $y=\bar{x}$ and that at $y=\underline{x}$, based on the physical consideration that the process is immediately and irreversibly transported $y=\bar{x}$ from to $y=\underline{x}$. In this research, we extend their approach to the model with imperfect interventions.

Now, we derive the NL-FPE based on a physical consideration. Since our focus in what follows is not the impulse control but rather the controlled population dynamics, we set $\bar{x}=1$ without any loss of generality. Set $\Omega=[0,1]$. The PDF $p$ is non-negative and defined in $\Omega$, and satisfies the conservation condition

$$
\int_{\Omega} p(y) \mathrm{d} y=1 \text {. }
$$

The flux $F=F(y)$ is assumed to belong to $C(\hat{\Omega})$ with $\hat{\Omega}=[0,1)$ and is defined as

$$
F(y)=\mu y p-\frac{\mathrm{d}}{\mathrm{d} y}\left(\frac{\sigma^{2} y^{2}}{2} p\right) \text {. }
$$

Set the left limit of $F$ at $y=1$ as

$$
\bar{F}=\lim _{y \rightarrow 1-0} F(y) .
$$

As in Yaegashi et al. [12], the following boundary conditions are specified:

$$
F(0)=0 \text { and } p(1)=0 \text {. }
$$

The first boundary condition is the usual one meaning that the process $\left(X_{t}\right)_{t \geq 0}$ is nonnegative and never approaches the boundary $y=0$ if it has a positive initial condition. The second one means that the process $\left(X_{t}\right)_{t \geq 0}$ is immediately absorbed at the boundary $y=1$, after that it is irreversibly transported to somewhere inside $\Omega$ according to the uncertainty $Z$.

What we have to find is the probabilistic relationship between the process $\left(X_{t}\right)_{t \geq 0}$ just before and after each intervention. From a physical consideration, the flux $\bar{F}$ is distributed in the interval $[a, b] \subset \subset \Omega$ according to the law $q$ as

$$
S(y)=\bar{F} q(y), a \leq y \leq b,
$$

where $S(y)$ is the source of probability increase per unit length at $y$. In this way, the total distributed probability from the boundary $y=1$ to the inside $\Omega$ is evaluated as

$$
\int_{a}^{b} S(y) \mathrm{d} y=\int_{a}^{b} \bar{F} q(y) \mathrm{d} y=\bar{F} \int_{a}^{b} q(y) \mathrm{d} y=\bar{F},
$$


meaning that this non-local treatment of the transport of probability is conservative. Note that $\bar{F}$ is a constant and $\int_{a}^{b} q(y) \mathrm{d} y=1$.

Considering the non-local relationship (8) combined with the standard derivation procedure for the other part of the domain, we get the complete description of the NLFPE as a non-local two-point boundary value problem:

$$
\frac{\mathrm{d} F}{\mathrm{~d} y}=\chi_{[a, b]} q(y) \bar{F} \text { inside } \Omega \text {, subject to (4) and (7). }
$$

Here, $\chi_{[a, b]}=1$ if $y \in[a, b]$ and $\chi_{[a, b]}=0$ otherwise. This kind of non-locality through the boundary flux is not common in the conventional non-local diffusive transport problems. Our goal is to solve the NL-FPE, which is achieved exactly for a simplified case, and is carried out numerically for general cases.

The Exact Solution. For analytical tractability, we consider the uniform distribution

$$
q(z)=\frac{1}{b-a}(a \leq z \leq b) .
$$

We briefly explain the derivation procedure of $p$ in this case. Set $r=\mu-\sigma^{2}>0$ and $D=\sigma^{2} / 2$. Firstly, the NL-FPE (10) is solved for $0 \leq y<a$ as

$$
p(y)=C_{1} y^{m}
$$

with $m=r / D$ and an unknown constant $C_{1}$. Secondly, in $a \leq y \leq b,(10)$ is solved as

$$
p(y)=C_{2} y^{m}-\frac{a \bar{F}}{(r+D)(b-a)} y^{-1}+\frac{\bar{F}}{r(b-a)}
$$

with an unknown constant $C_{2}$. Thirdly, (10) is solved in $b<y<1$ as

$$
p(y)=C_{3} y^{m}+\frac{\bar{F}}{r+D} y^{-1}
$$

with an unknown constant $C_{3}$.

We have four unknown constants $\bar{F}, C_{1}, C_{2}$, and $C_{3}$, meaning that we have to impose four conditions to completely determine them. We explore a continuous solution $p$ over the domain $\Omega$. Then, we impose the three conditions

$$
p(a-0)=p(a+0), p(b-0)=p(b+0), p(1)=0
$$

and the remaining one is the conservation condition (4). The four conditions are independent with each other, and give a unique tetrad $\left(\bar{F}, C_{1}, C_{2}, C_{3}\right)$ whose representations are not presented here because of page limitations. Consequently, we get

$$
p(y)= \begin{cases}C_{1} y^{m} & (0 \leq y<a) \\ C_{2} y^{m}-\frac{a \bar{F}}{(r+D)(b-a)} y^{-1}+\frac{\bar{F}}{r(b-a)} & (a \leq y \leq b) \\ \frac{\bar{F}}{r+D}\left(y^{-1}-y^{m}\right) & (b<y \leq 1)\end{cases}
$$


and

$$
F(y)= \begin{cases}0 & (0 \leq y<a) \\ \frac{y-a}{b-a} \bar{F} & (a \leq y \leq b) . \\ \bar{F} & (b<y<1)\end{cases}
$$

By uniqueness of the construction method of the PDF $p$, we get the proposition below.

Proposition 1. The NL-FPE (10) admits a unique continuous solution complying with the conservation condition (4). In addition, the flux $F$ is continuous inside $\Omega$.

The exact solution has the regularity $C([0,1]) \cap C^{2}((0, a) \cup(a, b) \cup(b, 1))$. Notice that the PDF of the perfect model [12] is derived from (16) under the limit $b \rightarrow a+0$, meaning that the present model considering the uncertainty is truly a generalization of the previous one. This solution is validated in the next section through a comparison with numerical solutions generated by a Monte-Carlo method and a finite volume scheme.

\section{$3 \quad$ Numerical Computation}

\subsection{Finite Volume Scheme}

Discretization. The finite volume scheme developed here is a generalization of the previous one [12]. The discretization starts from a time-dependent NL-FPE

$$
\frac{\partial p}{\partial t}+\frac{\partial F}{\partial y}=\chi_{[a, b]} q(y) \bar{F} \text { for } t>0 \text { and inside } \Omega \text {, subject to (7), }
$$

integrated from an initial condition satisfying the conservation condition (4) and identifies the stationary PDF $p$ with a steady solution to (18), which is numerically obtained with a sufficiently large $t>0$ in (18).

The domain $\Omega=[0,1]$ is divided into $N$ cells and $N+1$ nodes $y_{i}$ as

$$
0=y_{0}<y_{1}<\ldots<y_{L}<. .<y_{R}<\ldots<y_{N-1}<y_{N}=1
$$

so that $y_{L}=a$ and $y_{R}=b$. The nodes are located at the center of cells except at the boundaries $y=0,1$. For the sake of brevity, we assume the uniform discretization where the length between the nodes $\Delta y$ are uniform: $\Delta y=1 / N$. The time increment for temporal integration is denoted as $\Delta t$. The PDF $p$ approximated at the node $i$ and the time step $n$ is denoted as $p_{i}^{n}$. For the sake of brevity, set $y_{-1}=0$.

The semi-discretized (18) in the cell $i \quad(1 \leq i \leq N-1)$ is denoted as

$$
\frac{\mathrm{d} p_{i}}{\mathrm{~d} t}=-\frac{1}{\Delta x}\left(F_{i+\frac{1}{2}}-F_{i-\frac{1}{2}}\right)+S_{i},
$$

where $S_{i}$ corresponds to the discretized $S$ of (9) that is non-negative for $L \leq i \leq R$ and equals 0 otherwise. Each term of (20) is discretized as follows. 
Following the fitting technique that evaluates a numerical flux from exact solutions to auxiliary two-point boundary value problems $[12,16]$, the numerical flux $F_{i+\frac{1}{2}}$ is evaluated through exponential functions as

with

$$
F_{i+\frac{1}{2}}=-\frac{e^{\mathrm{Pe}}}{1-e^{\mathrm{Pe}}} V_{i+\frac{1}{2}} p_{i}+\frac{1}{1-e^{\mathrm{Pe}}} V_{i+\frac{1}{2}} p_{i+1}=\alpha_{i} p_{i}+\beta_{i} p_{i+1}
$$

$$
\mathrm{Pe}=\varepsilon_{i+\frac{1}{2}}^{-1} V_{i+\frac{1}{2}} \Delta y, V_{i+\frac{1}{2}}=\left(\mu-\sigma^{2}\right)\left(\frac{y_{i}+y_{i+1}}{2}\right), \varepsilon_{i+\frac{1}{2}}=D\left(\frac{y_{i}+y_{i+1}}{2}\right)^{2} .
$$

The source $S_{i}$ is constructed so that the discretized system is conservative. Set $K=R-L$ and discretize the domain $[a, b]$ of $q$ as

$$
a=z_{0}<z_{1}<\ldots<z_{K-1}<z_{K}=b .
$$

Set $z_{j+1 / 2}=\left(z_{j}+z_{j+1}\right) / 2$ and

$$
Q_{0}=\int_{z_{0}}^{z_{1 / 2}} q(z) \mathrm{d} z, Q_{j}=\int_{z_{j-1 / 2}}^{z_{j+1 / 2}} q(z) \mathrm{d} z(1 \leq j \leq K-1), Q_{K}=\int_{z_{K-1 / 2}}^{z_{K}} q(z) \mathrm{d} z,
$$

which are exactly or numerically evaluated so that

$$
\sum_{j=0}^{K} Q_{j}=1
$$

Then, $S_{i}$ is evaluated as

$$
S_{j+L}=F_{N-\frac{1}{2}} Q_{j}(0 \leq j \leq K) .
$$

Substituting (21) and (26) into (20) with an application of a fully-explicit discretization to the right-hand side yields

$$
\frac{p_{i}^{n+1}-p_{i}^{n}}{\Delta t}=-\frac{1}{\Delta y}\left(\alpha_{i} p_{i}^{n}+\beta_{i} p_{i+1}^{n}-\left(\alpha_{i-1} p_{i-1}^{n}+\beta_{i-1} p_{i}^{n}\right)\right)+S_{i}^{n} .
$$

Boundary conditions have to be specified to complete the discretization. At the boundary $y=0(i=0)$, we solve

$$
\frac{p_{0}^{n+1}-p_{0}^{n}}{\Delta t}=-\frac{2}{\Delta y}\left(\alpha_{0} p_{0}^{n}+\beta_{0} p_{1}^{n}\right),
$$

which is (27) with the boundary condition $F_{-\frac{1}{2}}=0$. At the boundary $y=1(i=N)$,

$$
p_{N}^{n}=0
$$

is directly specified. The system of linear equations containing (27), (28), and (29) is temporally evolved until the convergence condition

$$
\max _{i}\left|p_{i}^{n+1}-p_{i}^{n}\right|<\omega
$$

is satisfied for some $n \geq 1$, where $\omega=10^{-12}$ in this paper. Then, $p_{i}^{n+1}(0 \leq i \leq N)$ is taken as a numerical approximation to the NL-FPE (10). 
Mathematical Properties. The present finite volume scheme has the several important mathematical properties. Firstly, the scheme is conservative in a discrete sense. Namely, if the initial condition satisfies

$$
\Delta y\left(\frac{1}{2}\left(p_{0}^{0}+p_{N}^{0}\right)+\sum_{i=1}^{N-1} p_{i}^{0}\right)=1,
$$

then

$$
\Delta y\left(\frac{1}{2}\left(p_{0}^{n}+p_{N}^{n}\right)+\sum_{i=1}^{N-1} p_{i}^{n}\right)=1(n \geq 1),
$$

implying that numerical solutions are discrete analogue of the PDF. In addition, nonnegativity of the numerical solution is satisfied for sufficiently small $\Delta t$ [12], meaning that the scheme is stable. This stability is owing to the flux discretization (21). The scheme is simple and easy to implement, and furthermore conservative and stable as reviewed above. A drawback is that it may have at most first-order to second-order accurate, namely the error would decrease only linearly with respect to $\Delta y$ in the worst case. This drawback can be efficiently mitigated through the modern dual-meshing technique [17], which is currently undergoing by the authors. Notice that uniqueness of numerical solutions at each computation is clearly guaranteed because of using a fullyexplicit time discretization. An implicit discretization of our NL-FPE is also possible, but may be computationally inefficient because the coefficient matrix is dense.

\subsection{Numerical Tests}

The exact solution with a uniform distribution (11) is compared with numerical solutions generated with a standard Monte-Carlo method and the finite volume scheme. The Monte-Carlo method is based on a standard Euler-Maruyama discretization of the SDE(1) with the random numbers generated with Mersenne twisters. The total number of the sample points is $8 \times 10^{8}$ and the time increment for discretization of the SDE is 0.0001 . Unless otherwise specified, the computational resolution of the finite volume scheme is set as $\Delta y=1 / 600$ and $\Delta t=0.00005$ with $N=600, L=N / 3(a=1 / 3)$, and $R=2 N / 3(b=2 / 3)$. Considering the waterfowl management problem [7], the model parameters of the SDE are set as $\mu=0.17$ (1/year) and $\sigma=0.20$ ( $1 /$ year $^{1 / 2}$ ). These computational conditions are used unless otherwise specified. The initial condition is a delta distribution concentrated at $y_{50}$.

Fig. 2 demonstrates that the exact solution (16) and the two computational results agree well with each other, suggesting physical validity of the exact solution as a reasonable solution to the NL-FPE (10) and reasonable accuracy of the finite volume scheme. The convergence rate between the exact solution and numerical solutions with the scheme is then evaluated for different values of $\Delta y$. A four times finer temporal resolution was used for $N=1,200$ to maintain numerical stability. The computational results of the error are summarized in Table 1, suggesting second-order convergence of the scheme despite the scheme is linear and the NL-FPE (10) has an advection term that often provides a source of first-order error. The second-order convergence is 
checked from the quadratic decrease of the maximum nodal errors. This kind of attractive convergence property is called super-convergence [17], and the present scheme is thus super-convergent. The largest deviations between the exact and numerical solutions occur near the points $y=a, b$ where the regularity of $p$ decreases.

Finally, the scheme is applied to an advanced problem with $q(z)=(b-a) p((z-a) /(b-a))$, which leads to a nonlinear NL-FPE where the PDF $p$ modulates the uncertainty itself. This problem can be considered as the simplest model problem of a feed-back learning of the uncertainty. Theoretically, more realistic nonlinearity would be possible, but they will be addressed in our future work and the present numerical computation serves as its starting point. Fig. 3 shows the numerical solutions to the previous uniform $q$ and that with the present $p$-dependent one, demonstrating that the PDF of the latter clearly has a more concentrated profile. The conservation property (32) is correctly satisfied in this case despite its non-linearity and non-locality. Notice that this is a non-linear case that Monte-Carlo methods potentially become less efficient.

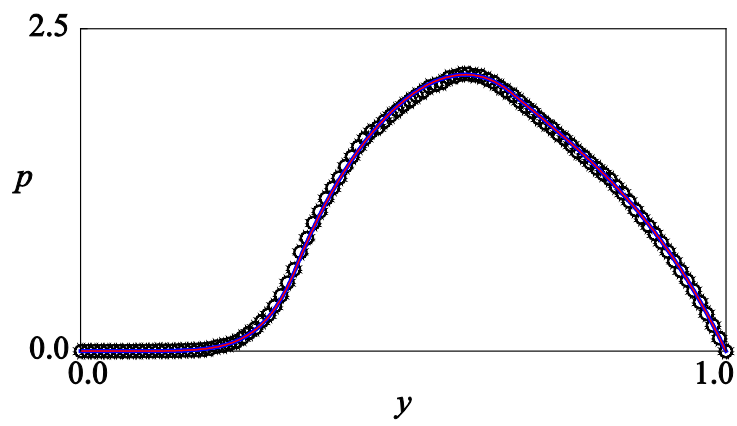

Fig. 2 Comparison of the exact solution (red line), a numerical solution generated by the MonteCarlo method (black circles), and a numerical solution by the finite volume scheme (blue line).

Table 1 The maximum nodal error of the finite volume scheme.

\begin{tabular}{rr}
\hline$N$ & Error \\
\hline 75 & $3.584 \mathrm{E}-03$ \\
150 & $8.988 \mathrm{E}-04$ \\
300 & $2.249 \mathrm{E}-04$ \\
600 & $5.623 \mathrm{E}-05$ \\
1,200 & $1.408 \mathrm{E}-05$ \\
\hline
\end{tabular}




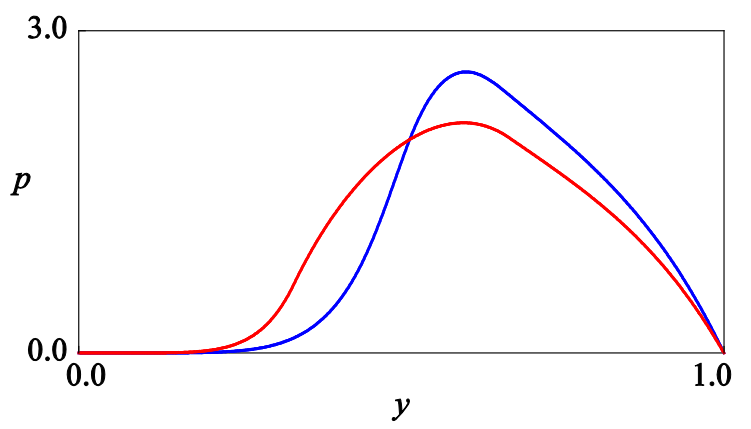

Fig. 3 Numerical solutions with the linear (red) and non-linear problems (blue).

\section{Conclusions}

The NL-FPE was derived for the first time and its analytical solution was found under a simplified condition. An explicit exponentially-fitted finite volume scheme was then presented to discretize the NL-FPE in a stable and conservative manner. The scheme successfully generated numerical solutions converging super-linearly toward the exact solution, demonstrating its satisfactory computational performance. Overall, we could efficiently and stably solve the non-local differential equation related to the impulse control by utilizing the sophisticated finite volume scheme.

A theoretical limitation of this work is that the underlying stochastic process is linear with respect to the state variable. This simplification is due to considering a tractable problem as a starting point of the present modeling framework of imperfect impulsive interventions, and the limitation can be abandoned without technical difficulties. More complex SDEs, like those having several state variables and/or jump noises, can be handled as well [18]. Loosely speaking, this extension can be achieved through formally adding appropriate terms to SDEs and NL-FPEs. The presented finite volume scheme then has to be extended so that the increased complexity of the corresponding NL-FPE is reasonably dealt with. Currently, we are considering an application of the present mathematical framework to ecosystem management involving the population of waterfowl and small migratory fish as its prey. This non-linear and multi-dimensional problem is theoretically of interest as well as practically of importance in fisheries resource management [19]. Deeper mathematical analysis of NL-FPEs, such as their solvability and regularity, is also currently undergoing.

\section{Acknowledgements}

JSPS KAKENHI 17J09125, 18K01714, and 19H03073 support this research. The first author engaged this research as a member of Fisheries Ecosystem Project Center of Shimane University. 


\section{References}

1. N’Guyen, A., Hirsch, P.E., Bozzuto, C., Adrian-Kalchhauser, I., Hôrková, K., BurkhardtHolm, P.: A dynamical model for invasive round goby populations reveals efficient and effective management options. J. Appl. Ecol. 55, 342-352 (2018).

2. Lysenko, D., Schott, S.: Food security and wildlife management in Nunavut. Ecol. Econ. 156, 360-374 (2019).

3. Øksendal, B.: Stochastic Differential Equations. Springer, Berlin, Heidelberg (2003).

4. Smith, V. L.: Control theory applied to natural and environmental resources an exposition. J. Environ. Econ. Manage. 4, 1-24 (1977).

5. Fleming, W. H., Soner, H. M.: Controlled Markov Processes and Viscosity Solutions. Springer, New York (2006).

6. Tang, S., Tang, G., Cheke, R.A.: Optimum timing for integrated pest management: modelling rates of pesticide application and natural enemy releases. J. Theor. Biol. 264, 623-638 (2010).

7. Yaegashi, Y., Yoshioka, H., Unami, K., Fujihara, M. A singular stochastic control model for sustainable population management of the fish-eating waterfowl Phalacrocorax carbo. J. Environ. Manage. 219, 18-27 (2018)

8. Yaegashi, Y., Yoshioka, H., Unami, K., Fujihara, M. A Stochastic impulse control model for population management of fish-eating bird Phalacrocorax Carbo and its numerical computation. In: Li L., Hasegawa K., Tanaka S. (eds.) Methods and Applications for Modeling and Simulation of Complex Systems. AsiaSim 2018. Communications in Computer and Information Science, vol 946, pp. 425-438. Springer, Singapore (2018).

9. da Costa Moraes, M. B., Nagano, M. S., Sobreiro, V. A.: Stochastic cash flow management models: A literature review since the 1980s. In: Guarnieri P. (eds.) Decision Models in Engineering and Management. Decision Engineering, pp. 11-28. Springer, Cham (2015).

10. Øksendal, B., Sulem, A.: Applied Stochastic Control of Jump Diffusions. Springer, Cham (2019).

11. Risken, H.: The Fokker-Planck Equation. Springer-Verlag, Berlin, Heidelberg (1996).

12. Yaegashi, Y., Yoshioka, H., Tsugihashi, K., Fujihara, M.: Analysis and computation of probability density functions for a 1-D impulsively controlled diffusion process. Comptes Rendus Mathematique 357, 306-315 (2019).

13. Cadenillas, A.: Optimal central bank intervention in the foreign exchange market, J. Econ. Theor. 87, 218-242 (1999).

14. Ohnishi, M., Tsujimura, M.: An impulse control of a geometric Brownian motion with quadratic costs. Eur. J. Oper. Res. 168, 311-321 (2006).

15. Evans, M. R., Majumdar, S. N.: Diffusion with optimal resetting. J. Phys. A: Math. Theor. 44(43). Paper No. 435001, 2011.

16. Yoshioka, H., Unami, K.: A cell-vertex finite volume scheme for solute transport equations in open channel networks. Prob. Eng. Mech. 31, 30-38 (2012).

17. Angermann, L., Wang, S.: A super-convergent unsymmetric finite volume method for convection-diffusion equations. J. Comput. Appl. Math. 358, 179-189 (2019).

18. Dang, D. M., Forsyth, P. A.: Better than pre-commitment mean-variance portfolio allocation strategies: A semi-self-financing Hamilton-Jacobi-Bellman equation approach. Euro. J. Oper. Res. 250, 827-841 (2016).

19. Yoshioka, H., Yaegashi, Y.: Mathematical analysis for management of released fish. Optim. Control Appl. Method. 39(2), 1141-1146 (2018). 